\title{
MODELOS DE CALIBRACIÓN PARA LA CUANTIFICACIÓN ESPECTROFOTOMÉTRICA DE HIDROCARBUROS TOTALES DEL PETRÓLEO EN SUELO
}

(Calibration models for the spectrophotometric quantification of total oil hydrocarbons in soil)

\author{
Nadia Viridiana ARGUIJO-PORTILLO ${ }^{1}$, Armando GUERRERO-PEÑA ${ }^{1 *}$, \\ Verónica Isidra DOMÍNGUEZ-RODRÍGUEZ², Eugenio CARRILLO-ÁVILA ${ }^{3}$ y Joel ZAVALA-CRUZ ${ }^{1}$
}

${ }^{1}$ Colegio de Postgraduados, Campus Tabasco, Periférico Carlos A. Molina s/n, km 3.5 carretera CárdenasHuimanguillo, 86500 H. Cárdenas, Tabasco, México

${ }^{2}$ División Académica de Ciencias Biológicas, Universidad Juárez Autónoma de Tabasco, km 0.5 carretera Villahermosa-Cárdenas, entronque Bosques de Saloya, 86150 Villahermosa, Tabasco, México

${ }^{3}$ Colegio de Postgraduados, Campus Campeche, km 17.5carretera Haltunchén-Edzná, Sihochac, 24450 Champotón, Campeche, México

*Autor para correspondencia: garmando@colpos.mx

(Recibido mayo 2017; aceptado agosto 2018)

Palabras clave: regresión lineal, contaminación, petróleo crudo

\section{RESUMEN}

Los modelos de calibración usados en química analítica deben ser previamente validados para cumplir con su objetivo, lo que generalmente se hace mediante análisis de regresión. En el presente estudio se proponen estrategias de validación interna y externa de modelos de calibración lineal simple (MCLS) para determinar hidrocarburos totales del petróleo (HTP) mediante espectrometría ultravioleta-visible (UV-Vis). En la validación cruzada, el modelo se emplea para estimar una a una las concentraciones de referencia con las que fue construido/calibrado, mientras que en la validación externa estima concentraciones de referencias que no fueron utilizadas en la calibración, con lo cual se demuestra su robustez. Se construyeron y validaron cuatro modelos, demostrando en todos los casos la linealidad y robustez de los MCLS en el rango estudiado. Los cuatro MCLS son adecuados para su aplicación en la cuantificación espectrofotométrica de HTP en suelos o en agua extraídos con diferentes disolventes, en cantidades que se encuentran dentro de los límites máximos permitidos en la normativa mexicana. En los modelos calibrados se identificó el efecto matricial debido al tipo de disolvente usado para diluir los hidrocarburos y por el tipo de hidrocarburo, lo cual influyó en los límites de detección y cuantificación.

Key words: linear regression, pollution, crude oil

\begin{abstract}
Calibration models used in analytical chemistry must be previously validated to achieve their aim. Generally, this is achieved by means of regression analysis. This study proposes strategies of internal and external validation of simple linear calibration models (LCM) to determine total petroleum hydrocarbons (TPH) by means of ultraviolet visible
\end{abstract}


spectrometry (UV-Vis). In the crossed validation, the model is used to estimate one by one the reference concentrations with which it was constructed/calibrated, whereas in the external validation the model estimates reference concentrations that were not used in its calibration, which demonstrates its robustness. Four models were constructed and validated, demonstrating in all cases the linearity and robustness of the MCLS in the studied range. All the models were adequate for the spectrometric quantification of TPH in soils or in water extracted by different solvents, in quantities that comply with the maximum permissible limits established by Mexican regulations. In the calibrated models, the matrix effect was identified due to the type of solvent used to dilute the hydrocarbons and the type of hydrocarbon, which is influenced by the limits of detection and quantification.

\section{INTRODUCCIÓN}

Los modelos analíticos de calibración basados en espectroscopia ultravioleta-visible (UV-Vis) para la determinación de los hidrocarburos totales del petróleo (HTP) en suelos y de hidrocarburos aromáticos (HA) en aceites vegetales, alimentos asados y agua, son una alternativa rápida para el análisis químico cuantitativo de muestras problema y han demostrado ser adecuados para este fin. La construcción de modelos de calibración lineal simple (MCLS) se lleva a cabo mediante una calibración en la que se emplean estándares del analito de interés y permite garantizar una relación fiable entre la cantidad de éste y una señal dada por el instrumento (Valcárcel y Cárdenas 2000). No debe confundirse con la calibración instrumental, la cual se realiza para asegurar el correcto funcionamiento del equipo empleando una sustancia diferente al analito de interés.

Con relación el método para la cuantificación de HTP en suelo, Wang et al. (2011) validaron un modelo lineal basándose en el coeficiente de correlación. A su vez, Dost e Ideli (2012) obtuvieron sus modelos de calibración mediante cromatografía líquida de alta resolución (HPLC) equipada con un detector UV-Vis. La linealidad de dichos modelos, desarrollados para la cuantificación de HA, fue evaluada por el método de mínimos cuadrados.

Si bien la mayoría de las técnicas convencionales de cuantificación de HTP se basan en el método gravimétrico por su sencillez, relativa rapidez y bajo costo, éste sólo es recomendable para suelos que contienen niveles superiores a $50000 \mathrm{mg} / \mathrm{kg}$ de HTP o de asfaltenos. En suelos con bajas concentraciones de hidrocarburos disminuye la precisión del método debido al error que implica el manejo de pesos pequeños (Fernández et al. 2006).

En estudios cuyo objetivo es el análisis cuantitativo o identificación de compuestos específicos en mezclas complejas de hidrocarburos como los aromáticos policíclicos, comúnmente se aplican técnicas como la cromatografía de gases (GC) o la HPLC, entre otras (Dost e Ideli 2012), pero estos métodos requieren largos tiempos de análisis por la necesidad de extracción de compuestos específicos, alto consumo de reactivos y elevado costo de los instrumentos.

Los modelos de calibración obtenidos por espectrofotometría son resultado de una calibración indirecta, ya que la cantidad del analito presente en las soluciones de referencia se determina en función de la señal dada por el instrumento analítico (CuadrosRodríguez et al. 2001).

En los métodos de calibración por UV-Vis reportados, las relaciones entre las concentraciones de referencia de hidrocarburos y la respuesta del espectrofotómetro son descritas por modelos lineales simples. Éstos, además de la estadística descriptiva, consideran como parámetros de validación de sus modelos de calibración el coeficiente de determinación $\left(\mathrm{R}^{2}\right)$, el coeficiente de correlación (r), los límites teóricos de detección y de cuantificación, y su precisión en condiciones de reproducibilidad (Wang et al. 2011, Dost e Ideli 2012).

Barwick (2003) establece paso a paso el proceso para la construcción de calibraciones, los parámetros estadísticos y la validación interna de los modelos de calibración, utilizando los siguientes indicadores: 1) el cálculo y representación gráfica de los residuales y 2) los estadísticos de la regresión, como r, , $\mathrm{R}^{2}$, la desviación estándar de los residuales o error estándar, el análisis de varianza (ANDEVA) y los coeficientes de regresión $m$ y a (pendiente y ordenada al origen), los cuales no son considerados en su totalidad en los estudios realizados por Vogt et al. (2000), Wang et al. (2011) y Dost e Ideli (2012).

Este estudio presenta una propuesta para la validación interna y externa de modelos de calibración 
lineal simple obtenidos por espectrofotometría UVVis, para determinar los HTP en suelos o en agua extraídos con diferentes disolventes y en niveles que no superan los límites máximos permitidos (3000 $\mathrm{mg} / \mathrm{kg}$ base seca de hidrocarburo de la fracción pesada, en suelos agrícolas) establecidos por la NOM138-SEMARNAT/SSA1-2012 (SEMARNAT 2013).

\section{MATERIALES Y MÉTODOS}

\section{Petróleos crudos y disolventes orgánicos}

Se utilizaron dos tipos de petróleo crudo, ligero (PCL) y pesado (PCP), proporcionados por Petróleo Mexicanos, cuyas densidades son similares a las reportadas por Santos et al. (2014): 0.83-0.87 y $0.92-1.0 \mathrm{~g} / \mathrm{cm}^{3}$, respectivamente. Las densidades correspondientes por grados del American Petroleum Institute (API) son de 31.1 a 39.0 y de 10.2 a $22.3^{\circ} \mathrm{API}$, respectivamente (IMP 2014).

Los disolventes empleados en la preparación de las soluciones de referencia o estándares de los petróleos crudos fueron hexano ultrapuro y éter etílico grado ACS. El primero tiene una polaridad más baja que el segundo con base en sus constantes dieléctricas: 1.9 y 4.3, respectivamente (Masschelein-Kleiner 2004).

\section{Espectrofotómetro UV-Vis}

Para las determinaciones se utilizó un espectrofotómetro UV-Vis marca Perkin Elmer, modelo Lambda 25 de doble haz, con un intervalo de escaneo de 200 a $1100 \mathrm{~nm}$. Los registros de los espectros fueron realizados cada $2 \mathrm{~nm}$ con una velocidad de escaneo de $240 \mathrm{~nm} / \mathrm{min}$.

\section{Análisis preliminar de un calibrado de petróleo} crudo ligero mediante $\mathrm{UV}$-Vis

En un análisis preliminar se obtuvo el espectro completo de la región UV-Vis (200 a $1100 \mathrm{~nm})$ de soluciones de referencia de concentraciones de 0 a $1000 \mathrm{mg} / \mathrm{L}$. Esto se realizó con la finalidad de encontrar una longitud de onda $(\lambda)$ en el espectro UV-Vis en la que se observara una respuesta lineal entre la concentración y la absorbancia.

Una vez que se obtuvo la $\lambda$ esperada, se procedió a desarrollar la metodología, aplicando dicha $\lambda$ para la obtención de los modelos con las combinaciones restantes de éter etílico y el PCP. Para esta etapa preliminar se prepararon 17 concentraciones (número mayor de siete, que es el mínimo indicado por Barwick [2003]) de $0,1,2,4,6,8,10,20,40,60,80,100,200,400,600$, 800 y $1000 \mathrm{mg} / \mathrm{L}$ de PCL. Las soluciones se prepararon en matraces volumétricos clase A de $25 \mathrm{~mL}$.
Las concentraciones de 0 a 10 , de 20 a 100 y de 200 a $1000 \mathrm{mg} / \mathrm{L}$ se prepararon a partir de soluciones stock de 10, 100 y 1000 de PCL, respectivamente, todas a un volumen final de $25 \mathrm{~mL}$ diluido con hexano (el disolvente).

Preparación y análisis por UV-Vis de los calibrados de petróleo crudo en dos diferentes disolventes

Al incluir los dos disolventes y los dos tipos de petróleo crudo en esta segunda etapa de la experimentación, se obtuvieron las siguientes combinaciones: 1) hexano-petróleo crudo ligero, 2) hexano-PCP, 3) éter-PCL y 4) éter-PCP. A partir de éstas se obtuvieron cuatro calibrados.

Cada calibrado se preparó con ocho concentraciones de referencia: 0, 4, 8, 20, 40, 60, 80 y $100 \mathrm{mg} / \mathrm{L}$ (el mínimo indicado por Barwick [2003] es siete), con cinco repeticiones de cada una para tener un total de 40 soluciones por calibrado.

La absorbancia de todas las soluciones (160 de los cuatro calibrados) se midió en un espectrómetro $\mathrm{UV}$-Vis a $254 \mathrm{~nm}$. En este valor de $\lambda$ se encontró la mejor correlación lineal entre la concentración de petróleo y la absorbancia, definida en la etapa preliminar. El ajuste del cero de absorbancia se realizó con agua desionizada.

\section{Validación de los modelos de calibración}

Para llevar a cabo la validación primero se dividieron en dos grupos los resultados de las absorbancias de las 40 soluciones de referencia de cada combinación. Para el primer grupo, denominado grupo de entrenamiento, sólo se tomaron los resultados de 35 soluciones, equivalentes al $90 \%$, y se utilizaron para la construcción del modelo mediante una regresión lineal simple de los puntos graficados con objeto de realizar la calibración o validación interna.

Para el segundo grupo, denominado grupo de validación, se tomaron las absorbancias de las cinco soluciones de referencia (equivalente al $10 \%$ de las soluciones) omitidas en el grupo de entrenamiento. Este grupo se utilizó para la validación externa del modelo.

\section{Validación interna}

La validación interna de los modelos de calibración se llevó a cabo aplicando los parámetros que se describen a continuación.

\section{Validación cruzada}

Se dividieron las absorbancias de las 35 soluciones de referencia del grupo de entrenamiento en 
35 grupos, de los cuales 34 se tomaron para establecer una primera calibración mediante regresión lineal simple. El otro grupo se tomó para la predicción o validación.

El proceso se repitió iterativamente hasta que cada una de las concentraciones de referencia fue predicha con el resto de los grupos de calibración.

\section{Coeficiente de determinación (R2)}

Se obtuvieron los $\mathrm{R}^{2}$ de los cuatro modelos de regresión de los resultados de las 35 soluciones de referencia correspondientes al grupo de entrenamiento, no así los de la validación cruzada.

\section{ANDEVA de la regresión}

Se realizó el ANDEVA de la regresión de las soluciones del grupo de entrenamiento. Se estableció la hipótesis nula de que la absorbancia $(y)$ no es una función de la concentración $(x)$, frente a la hipótesis alternativa de que $y$ sí está en función de las variaciones de $x$, aceptándose esta última si $F>F$ crítica. Al aceptarse la hipótesis alternativa se indica que el modelo lineal es significativo (Weisberg 2005).

\section{Residuales y error absoluto medio (EAM)}

Se presentan los gráficos de los residuales y los valores del EAM de los cuatro modelos.

\section{Coeficiente de correlación (r)}

Se propone utilizar $r$ para explicar el grado de asociación entre las concentraciones estimadas por dichos modelos y el conjunto de concentraciones de referencia correspondientes, para medir su precisión.

\section{Prueba t de Student}

Se propone realizar una prueba t de Student para datos apareados con el objeto de definir si existen o no diferencias estadísticas entre los mismos valores que se usaron para $r$.

La prueba t se realizó con una probabilidad del $5 \%$, con la hipótesis nula de que no existen diferencias estadísticas entre las concentraciones de referencia y las concentraciones estimadas por los modelos, frente a la hipótesis alternativa de que sí existe diferencia entre los valores de referencia y los estimados, aceptándose la primera si p-valor $>0.05$.

Los estadísticos empleados para la validación de los modelos se describen a continuación (Marqués 2010).

Coeficiente de determinación $\left(R^{2}\right)$ del modelo de regresión lineal

$R^{2}=(r)^{2}$ donde $r$ es el coeficiente de correlación para las variables de las curvas de calibración

$\mathrm{r}=\frac{\sum_{\mathrm{i}=1}^{\mathrm{n}}\left[\left(\mathrm{x}_{\mathrm{i}}-\overline{\mathrm{x}}\right)\left(\mathrm{y}_{\mathrm{i}}-\overline{\mathrm{y}}\right)\right.}{\left\{\left[\sum_{\mathrm{i}=1}^{\mathrm{n}}\left[\left(\mathrm{x}_{\mathrm{i}}-\overline{\mathrm{x}}\right)^{2}\right]\left[\sum_{\mathrm{i}=1}^{\mathrm{n}}\left(\mathrm{y}_{\mathrm{i}}-\overline{\mathrm{y}}\right)^{2}\right]\right\}^{1 / 2}\right.}$

donde $x_{\mathrm{i}}$ es el valor observado en el eje $\mathrm{x}, y_{\mathrm{i}}$ el valor observado en el eje y, la media de los valores $x_{\mathrm{i}}$; la media de los valores $y_{\text {i, }}$ y $n$ el número de puntos de calibración.

Residuales $\left(e_{i}\right)$ y error absoluto medio EAM

$e_{i}=y_{i}-\bar{y}_{i}$

$\mathrm{EAM}=\frac{\sum_{i=1}^{n}\left|e_{i}\right|}{n}$

Análisis de varianza (ANDEVA) de la regresión de las curvas de calibración

Suma de cuadrados de los residuales:

$S C_{\text {residuales }}=\sum_{i=1}^{n}\left(e_{i}\right)^{2}=\sum_{i=1}^{n}\left(y_{i}-\bar{y}_{i}\right)^{2}$

Suma de cuadrados de la regresión:

$S C_{\text {regresión }}=\sum_{i=1}^{n}\left(y_{i}-\bar{y}\right)^{2}$

Pendiente $(m)$ de la línea de regresión:

$m=\frac{\sum_{i=1}^{n}\left[\left(x_{i}-\bar{x}\right)\left(y_{i}-\bar{y}\right)\right.}{\sum_{i=1}^{n}\left[\left(x_{i}-\bar{x}\right)^{2}\right.}$

Ordenada al origen $(a)$ de la línea de regresión

$a=\bar{y}-m \bar{x}$

donde $=$ valores de $y$ estimados por la ecuación de regresión para un valor dado de $x$.

Prueba t de Student para datos apareados entre concentraciones estimadas por los modelos y las concentraciones de referencia

$t=\frac{\left(\bar{x}_{d}\right)(\sqrt{n})}{s_{d}}$

donde $\bar{x}_{d}$ es la media de las diferencias de los valores de las variables, $s_{d}$ el número total de diferencias y la desviación estándar de las diferencias de los valores de las variables. 


\section{Validación externa}

Durante la validación externa se estimaron las cinco concentraciones de referencia del grupo de validación utilizando el modelo construido con las 35 concentraciones del grupo de entrenamiento durante la validación interna. De esta manera se tuvo un conjunto de concentraciones de referencia y otro de concentraciones estimadas, para cada modelo de calibración.

\section{Coeficiente de correlación (r)}

En la validación externa se calculó el valor de $r$ para establecer el grado de asociación entre las concentraciones estimadas y las concentraciones de referencia del conjunto del $10 \%$ de las mediciones o grupo de validación.

\section{Prueba t de Student}

Para el cálculo del estadístico t de Student para datos apareados se aplicaron las mismas hipótesis y criterios de rechazo y aceptación que para la validación interna.

\section{Límites de detección y cuantificación de los cuatro modelos de calibración}

Se calcularon los límites de detección y de cuantificación de cada modelo. Primero se obtuvo la media del conjunto de concentraciones del blanco (concentración $0 \mathrm{mg} / \mathrm{L}$ ) estimadas por los modelos. El límite de detección se obtuvo sumando a la media de las concentraciones del blanco, tres veces su desviación estándar.

El límite de cuantificación se calculó sumando a la media de las concentraciones del blanco 10 veces su desviación estándar (Shrivastava y Gupta 2011).

\section{RESULTADOS Y DISCUSIÓN}

\section{Análisis preliminar de un calibrado de petróleo crudo mediante espectrofotometría}

La obtención del espectro completo de la región UV-Vis (Fig. 1) de las soluciones de referencia (0 a $1000 \mathrm{mg} / \mathrm{L}$ ) de PCL en hexano, permitió identificar que en la $\lambda$ de $254 \mathrm{~nm}$ las soluciones presentan un pico de absorción con respuesta lineal entre la concentración de PCL y la absorbancia. Este resultado fue ratificado repitiendo la medición de dichas soluciones, pero únicamente a una $\lambda$ de 254 $\mathrm{nm}$, también en el intervalo de concentración de 0 a $1000 \mathrm{mg} / \mathrm{L}$ de PCL. A partir de las absorbancias obtenidas del segundo análisis se construyó el gráfico con la recta ajustada por regresión, la cual se muestra en la figura 2.

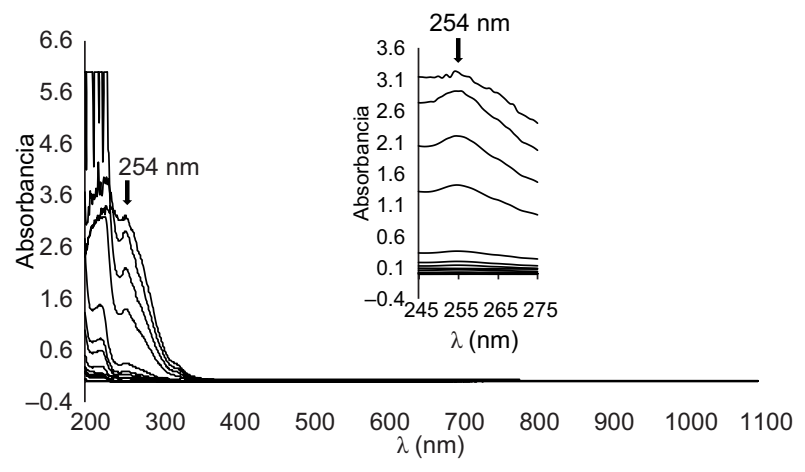

Fig. 1. Espectro completo de absorbancia de la región UV-Vis para el intervalo de concentración de 0 a $1000 \mathrm{mg} / \mathrm{L}$ en las concentraciones de referencia de petróleo crudo ligero (PCL) en hexano, y ampliación de la región de $254 \mathrm{~nm}$

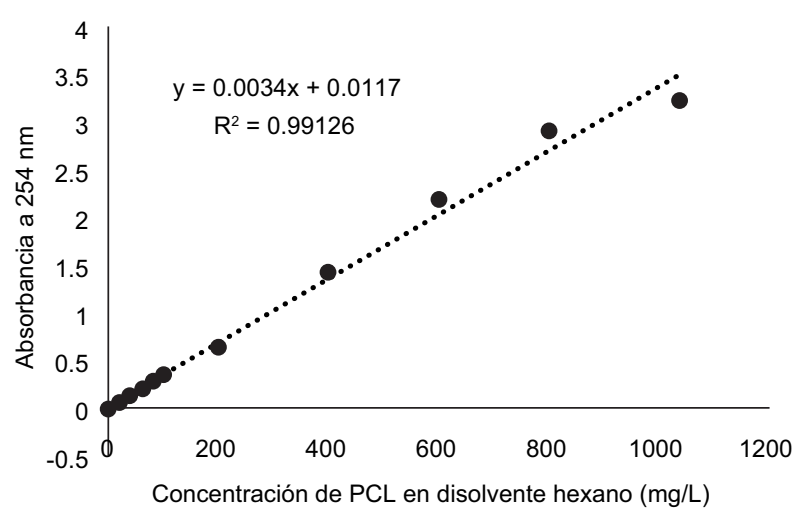

Fig. 2. Ajuste de las absorbancias de las concentraciones de petróleo crudo ligero (PCL) en hexano en el intervalo de concentración de 0 a $1000 \mathrm{mg} / \mathrm{L}$, a la recta ajustada mediante regresión lineal de las soluciones de referencia, medidas a $254 \mathrm{~nm}$

La $\lambda$ de $254 \mathrm{~nm}$ seleccionada en el presente estudio coincide con la reportada por Dost e Ideli (2012), quienes observaron que es la mejor respuesta lineal para nueve hidrocarburos aromáticos policíclicos (HAP) en la región UV. Por su parte, Wang et al. (2011) observaron una absorción estable a un valor de $\lambda$ de 230 y $200 \mathrm{~nm}$ para una mezcla de hidrocarburos disueltos en diclorometano y hexano, respectivamente.

Preparación y análisis por UV-Vis de los calibrados de petróleo crudo en diferentes disolventes

En la figura 3 se presentan los gráficos de las cuatro curvas de calibración, con cinco repeticiones para cada concentración de los petróleos crudos. La representación gráfica de los puntos de calibración (absorbancia-concentración de los petróleos 


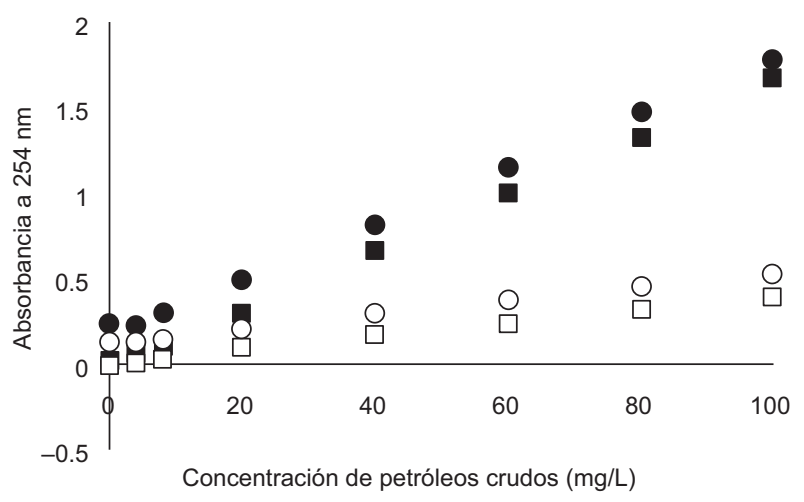

$\square$ Hexano-PL OÉter-PL

Hexano-PP Éter-PP

Fig. 3. Curvas de calibración de las concentraciones de referencia de los petróleos crudos en el intervalo de 0 a 100 $\mathrm{mg} / \mathrm{L}$, en dos disolventes. PCL: petróleo crudo ligero, PCP: petróleo crudo pesado

crudos) es una opción rápida para observar el comportamiento de los datos en cuanto a linealidad, y constituye una forma cualitativa de evaluarla.
También se observa el efecto de la combinación de disolventes-petróleos crudos en las diferentes pendientes $(m)$ de cada caso.

Las absorbancias de los calibrados de PCL resultaron menores debido a la baja densidad de éste respecto del PCP, cuyas absorbancias fueron mayores. Además, el PCL contiene hidrocarburos de menor peso molecular, a diferencia del PCP. Todo esto influyó también en las variaciones observadas entre las $m$ de los modelos ambos petróleos.

\section{Validación de los modelos}

Validación interna o calibración

Coeficiente de determinación $\left(R^{2}\right)$ del modelo de regresión lineal

Como resultado del proceso de validación interna se construyeron cuatro modelos lineales entre la absorbancia a $254 \mathrm{~nm}$ y las concentraciones de petróleos crudos (Fig. 4) con valores de $\mathrm{R}^{2}$ de 0.9952 a 0.9996. Esto significa que los modelos explican en promedio el $99.74 \%$ de la variabilidad de los resultados. Valores de $\mathrm{R}^{2}$ similares fueron obtenidos por Dost e Ideli (2012) en la validación de un método de detección de hidrocarburos aromáticos policíclicos (HAP) por
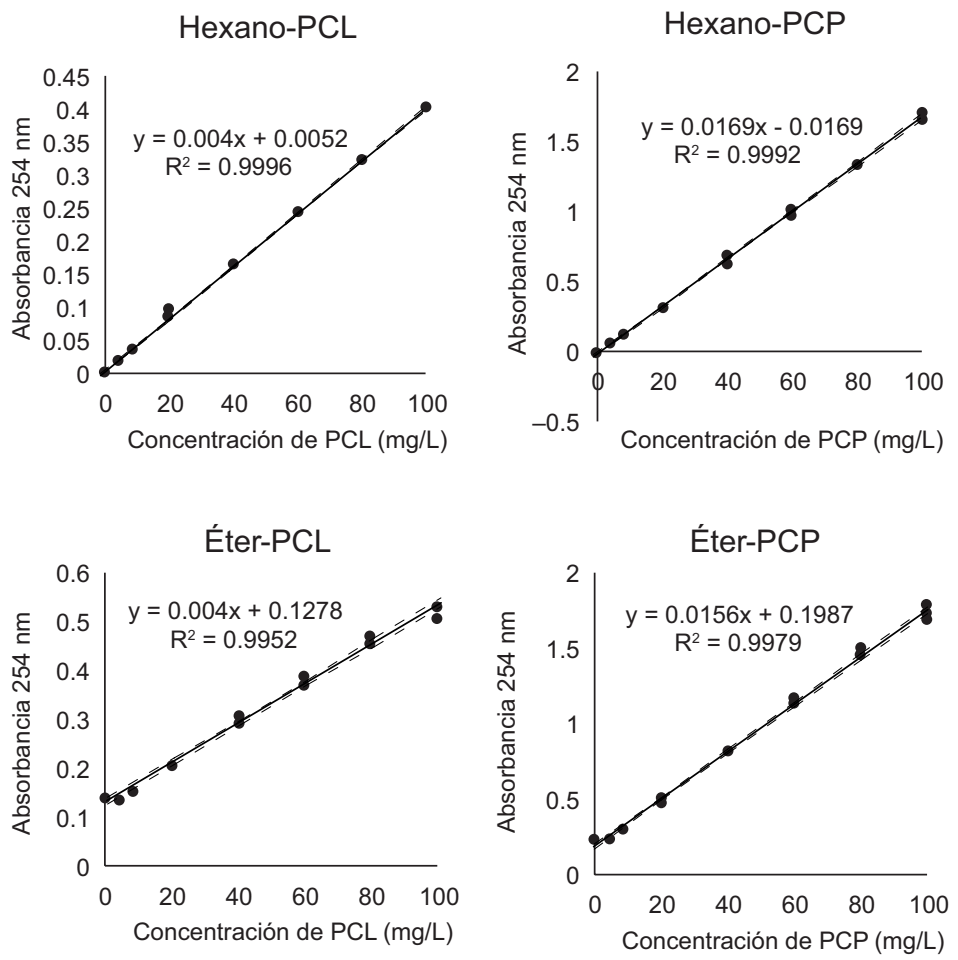

Fig. 4. Modelos de calibración y coeficientes de determinación obtenidos por el ajuste de las absorbancias experimentales a la recta ajustada mediante regresión lineal simple para la validación interna, con bandas de confianza al $99 \%$ de probabilidad. PCL: petróleo crudo ligero, PCP: petróleo crudo pesado 
HPLC/UV-Vis; y por Wang et al. (2011) en mezclas de hidrocarburos de petróleo.

En la figura 4 se observa también que para los modelos de calibración con PCL y PCP, el disolvente usado influye mediante la modificación de los valores de $a$ en las ecuaciones de regresión, siendo en ambos casos mayores con éter etílico que con hexano. Las variaciones en los valores de $a$ pueden atribuirse a la densidad de los disolventes, ya que el éter etílico es más denso que el hexano $\left(713.4 \mathrm{~kg} / \mathrm{m}^{3}\right.$ y $654.8 \mathrm{~kg} /$ $\mathrm{m}^{3}$, respectivamente).

Por otra parte, los valores de $m$ son influenciados por el tipo de hidrocarburo, siendo mayores con el PCP en ambos disolventes. En el caso de los modelos hechos con PCL, $m$ conserva su valor indistintamente del disolvente usado (0.004) y en los modelos hechos con PCP, $m$ mostró un ligero aumento con el hexano respecto al éter etílico (0.0169 y 0.0156 , respectivamente). Aun así, son valores muy cercanos entre sí.

Los cambios en los valores de $m$ en las curvas debidos al tipo de petróleo se pueden explicar por las características físicas de cada uno, específicamente por su densidad. El PCP es más denso que el PCL, por lo que las absorbancias fueron mayores para los calibrados con PCP, llegando hasta 1.7 a $100 \mathrm{mg} / \mathrm{L}$, mientras que los calibrados con PCL mostraron una absorbancia de 0.5 a la misma concentración.

Al tratarse de cuatro combinaciones de disolvente-petróleo crudo preparados en condiciones e intervalos de concentración estándar iguales, las variaciones observadas en las absorbancias evidencian la existencia de efectos matriciales por la composición de las soluciones, mismas que afectan directamente los valores de los coeficientes de los modelos. El efecto matricial en los modelos de calibración se descarta al ajustar el cero de absorbancia empleando el blanco de reactivos de los dos disolventes usados.

Danzer y Currie (1998) afirman que el efecto matricial de las muestras problema puede contrarrestarse utilizando el método de adición de estándar, el cual consiste en adicionar soluciones estándar a la muestra para crear un comportamiento similar del conjunto de calibración y de la muestra siempre que el analito se añada en la forma de la misma especie.

En la figura 4 se observa que el intervalo de confianza (IC) al $99 \%$ (región enmarcada por las bandas punteadas a ambos lados de la recta ajustada) es muy estrecho para las cuatro líneas ajustadas, especialmente para los calibrados en que se usó hexano como disolvente, lo cual coincide con los bajos valores de los residuales. Estos resultados son congruentes con los conceptos de IC reportados por Newcombe (1998) y Barwick (2003).

\section{ANDEVA de la regresión}

El análisis de varianza es considerado un método no gráfico para evaluar la linealidad de un modelo de calibración (Raposo 2016). El ANDEVA de los modelos de regresión de los cuatro calibrados (Cuadro I) muestra que las sumas de cuadrados de las regresiones son superiores a la suma de cuadrados de los residuales en todos los casos. Para magnificar estas diferencias se tiene el valor del estadístico $\mathrm{F}$, que resultó significativamente mayor que su valor crítico, lo cual es indicativo de que los cuatro modelos son significativamente lineales.

El modelo del calibrado hexano-PCL es capaz de describir el $99.96 \%$ de la variabilidad de los datos, el del hexano-PCP el 99.92\%, el del éter-PCL el 99.52 $\%$ y el del éter-PCP el $99.79 \%$.

\section{Residuales y EAM}

De acuerdo con la figura 5, la distribución de los residuales indica un comportamiento más aleatorio para el calibrado hexano-PCL que para el resto, destacando además que el intervalo de dichos valores fue el más cercano a cero $(0.0122 \mathrm{a}-0.0033)$.

Los intervalos correspondientes a los residuales de los calibrados éter-PCL, hexano-PCP y éter-PCP presentaron una dispersión respecto al cero del orden siguiente: $0.0209 \mathrm{a}-0.0215,0.0450 \mathrm{a}-0.0359$ y 0.0557 a -0.0663 , respectivamente.

El EAM o promedio de los valores absolutos de los residuales se cataloga como una medida de desviación (Mayer y Butler 1993) aplicada en la validación de modelos de calibración. Los valores para los calibrados hexano-PCL, hexano-PCP, éter-PCL y éter-PCP fueron $0.0017,0.0133,0.0081$ y 0.0214 , respectivamente.

Prueba $t$ de Student para datos apareados entre concentraciones estimadas y concentraciones de referencia

Los resultados de la prueba $t$ de Student para datos apareados o relacionados mostraron que no existió diferencia significativa $(\mathrm{p}>0.05)$ entre las concentraciones estimadas por los modelos y las concentraciones de referencia de las muestras del grupo de entrenamiento, en los cuatro modelos analizados.

Coeficiente de correlación (r) entre concentraciones estimadas y concentraciones de referencia

Los valores de $r$ entre las concentraciones estimadas y las de referencia (Fig. 6) obtenidos en 
CUADRO I. ANDEVA DE LOS CUATRO MODELOS DE CALIBRACIÓN

\begin{tabular}{|c|c|c|c|c|c|}
\hline \multicolumn{6}{|c|}{ Hexano-PCL } \\
\hline & $\begin{array}{l}\text { Grados de } \\
\text { libertad }\end{array}$ & $\begin{array}{l}\text { Suma de } \\
\text { cuadrados }\end{array}$ & $\begin{array}{l}\text { Promedio de } \\
\text { los cuadrados }\end{array}$ & $\mathrm{F}$ & $\begin{array}{c}\text { Valor crítico } \\
\text { de F }\end{array}$ \\
\hline Regresión & 1 & 0.71846936 & 0.71846936 & 86096.4487 & 5.8204E-58 \\
\hline Residuos & 33 & 0.00027538 & 8.3449E-06 & & \\
\hline Total & 34 & 0.71874474 & & & \\
\hline \multicolumn{6}{|c|}{ Hexano-PCP } \\
\hline Regresión & 1 & 12.0796147 & 12.0796147 & 42363.2316 & $6.9845 \mathrm{E}-53$ \\
\hline Residuos & 33 & 0.00940975 & 0.00028514 & & \\
\hline Total & 34 & 12.0890244 & & & \\
\hline \multicolumn{6}{|c|}{ Éter-PCL } \\
\hline Regresión & 1 & 0.73864744 & 0.73864744 & 6878.49859 & $6.9648 \mathrm{E}-40$ \\
\hline Residuos & 33 & 0.0035437 & 0.00010738 & & \\
\hline Total & 34 & 0.74219114 & & & \\
\hline \multicolumn{6}{|c|}{ Éter-PCP } \\
\hline Regresión & 1 & 11.2470541 & 11.2470541 & 15714.2794 & $8.738 \mathrm{E}-46$ \\
\hline Residuos & 33 & 0.02361882 & 0.00071572 & & \\
\hline Total & 34 & 11.270673 & & & \\
\hline
\end{tabular}

PCL: petróleo crudo ligero, PCP: petróleo crudo pesado
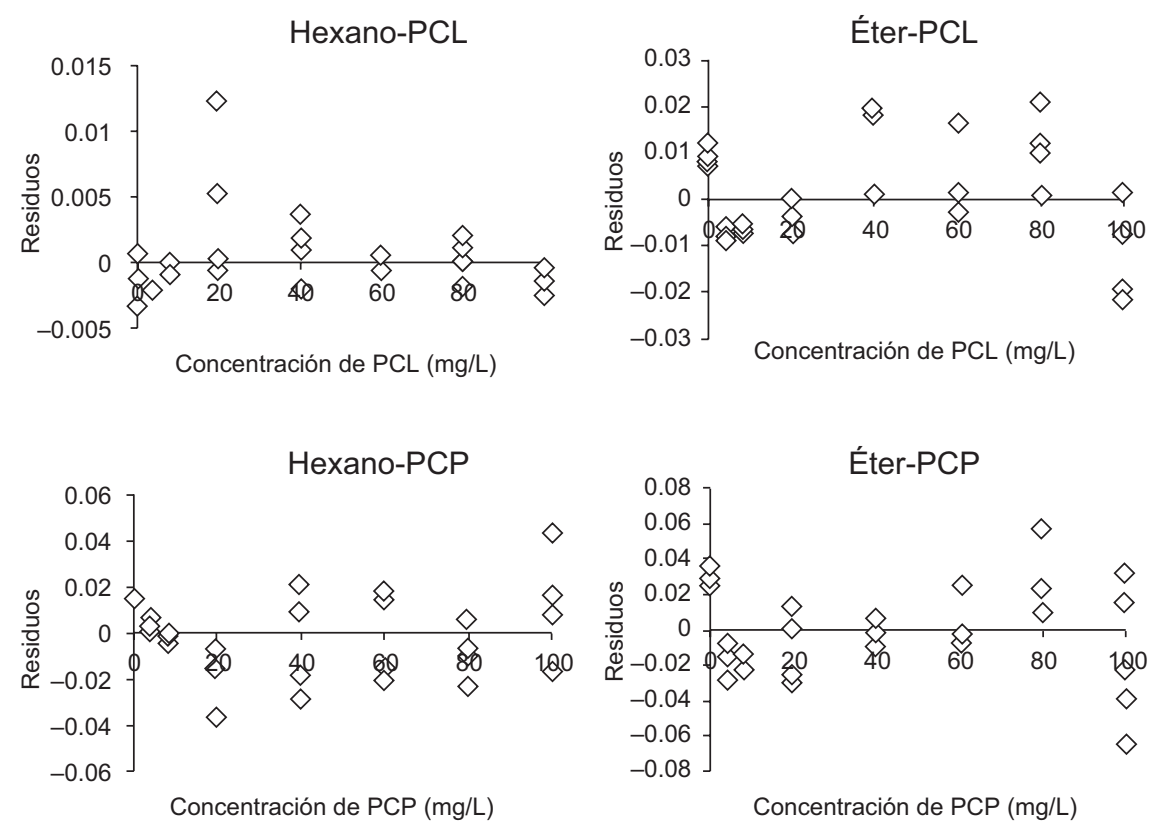

Fig. 5. Gráficos de residuales de las regresiones lineales de los cuatro calibrados para la validación interna de los modelos. PCL: petróleo crudo ligero, PCP: petróleo crudo pesado 

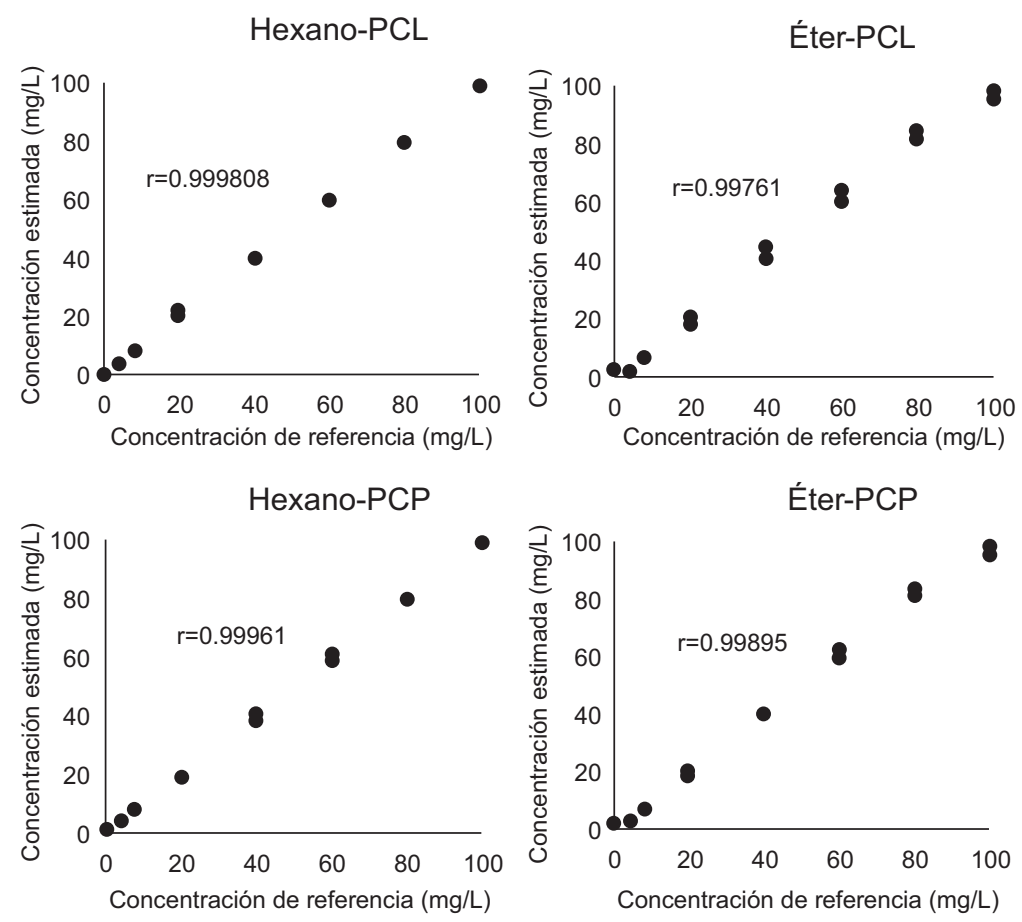

Fig. 6. Coeficientes de correlación entre las muestras observadas y estimadas, obtenidos durante la validación interna de los modelos de calibración. PCL: petróleo crudo ligero, PCP: petróleo crudo pesado

la validación interna, fueron superiores a 0.9996 para los calibrados hexano-PCL y hexano-PCP, y en el caso de los calibrados éter-PCL y éter-PCP fueron superiores a 0.9976 . En todos los casos estos resultados indican una alta asociación entre dichas concentraciones como lo indica el valor cercano a 1. El uso de r como medida de la linealidad del modelo es ampliamente reconocido (Barwick 2003, Raposo 2016). La correlación entre las variables analizadas puede ser o no lineal (van Loco et al. 2002).

\section{Validación externa de los modelos de calibración} Prueba t de Student para datos apareados

Con la prueba $t$ de Student se encontró que no existió diferencia estadística $(\mathrm{p}>0.05)$ entre las medias de las concentraciones estimadas por los modelos y las concentraciones de referencia del conjunto del $10 \%$ de las mediciones que se omitieron en la construcción y calibración de los modelos.

Coeficiente de correlación (r) entre concentraciones estimadas y concentraciones de referencia

Los valores de r obtenidos en la validación externa (Fig. 7) para las concentraciones estimadas con los modelos y las concentraciones de referencia del grupo de validación, fueron superiores a 0.9993 para los calibrados hexano-PCL, hexano-PCP y éter-PCP, e igual a 0.9983 para el calibrado éter-PCL. En todos los casos existe un alto grado de asociación entre ambos conjuntos de concentraciones por los valores cercanos a 1 .

\section{Límites de detección y cuantificación de los ca- librados}

Una vez validados los modelos se calcularon los límites de detección y los límites de cuantificación a partir de los datos obtenidos con los calibrados, para cada uno de los modelos de calibración. Dichos límites se muestran en el cuadro II, que también incluye las medias y desviaciones estándar de los blancos.

Los valores más bajos en el límite de detección y de cuantificación correspondieron a ambos tipos de petróleo crudo con hexano, mientras que para los calibrados con éter fueron mayores. Este resultado coincide con los valores de las ordenadas al origen en los modelos mostrados en la figura 4.

En contraste, los valores encontrados para modelos generados para HAP utilizando concentraciones estándares de HA (Dost e Ideli 2012) y los límites de detección para HA (Vogt et al. 2000) fueron menores a los de este estudio. La diferencia en dichos valores respecto a los encontrados en este trabajo puede 

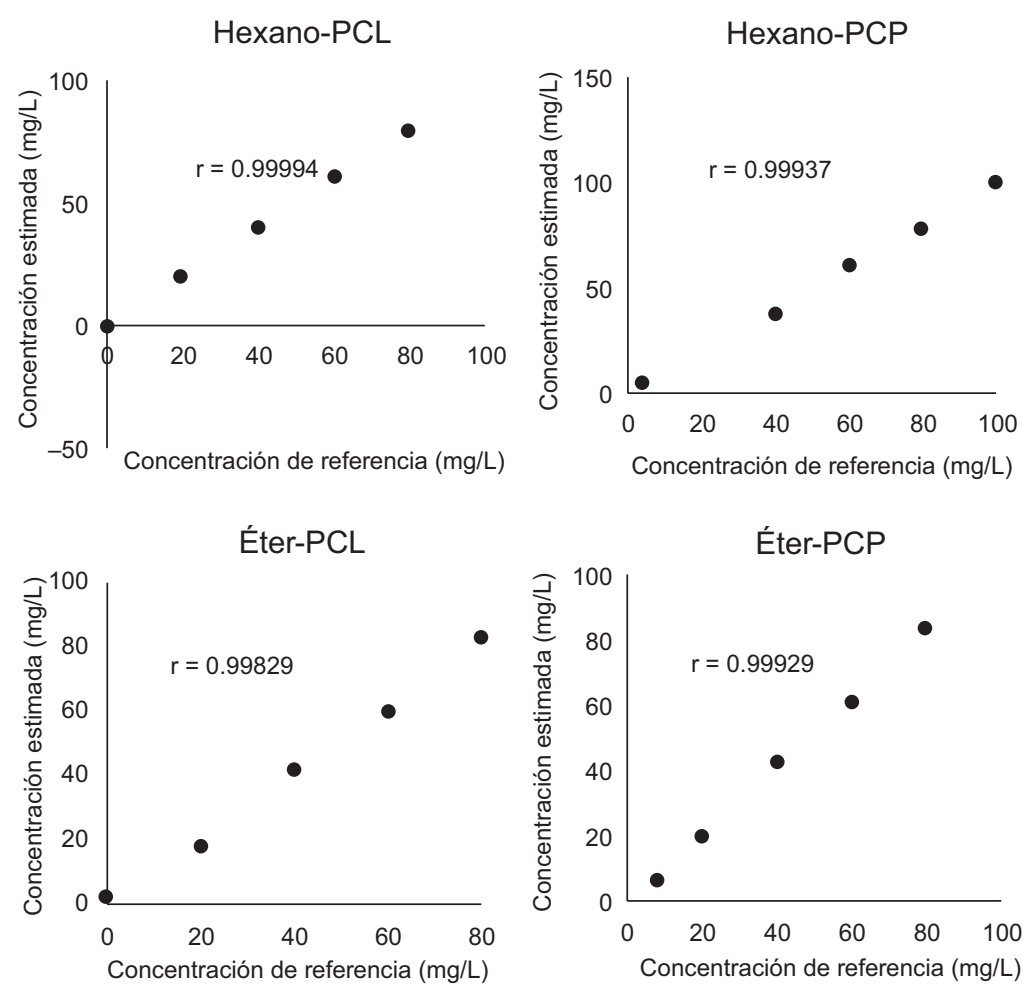

Fig. 7. Coeficientes de correlación obtenidos durante la validación externa de los cuatro modelos de calibración. PCL: petróleo crudo ligero, PCP: petróleo crudo pesado

CUADRO II. LÍMITES DE DETECCIÓN Y CUANTIFICACIÓN PARA LOS MODELOS DE CALIBRACIÓN

\begin{tabular}{lcccc}
\hline & Hexano-PCL & Hexano-PCP & Éter-PCL & Éter-PCP \\
\hline Límite de detección (mg/L) & 0.82 & 1.03 & 3.70 & 2.81 \\
Límite de cuantificación (mg/L) & 3.72 & 1.21 & 6.98 & 4.7 \\
Media de los valores estimados del blanco & -0.425 & 0.9556 & 2.3 & 1.9743 \\
DE de los valores estimados del blanco & 0.4145 & 0.0256 & 0.4677 & 0.2775 \\
\hline
\end{tabular}

PCL: petróleo crudo ligero, PCP: petróleo crudo pesado, DE: desviación estándar

deberse a que los autores emplearon compuestos puros para sus análisis y en este trabajo se emplearon petróleos crudos cuya composición es más compleja.

\section{CONCLUSIÓN}

La evaluación interna y externa de los MCLS propuesta para calificar las calibraciones por espectrofotometría UV-Vis indican que las estimaciones para cuantificar HTP en suelos o en agua, extraído con diferentes disolventes, son adecuadas para su aplicación en concentraciones menores a las esta- blecidas como límites máximos permitidos en la normativa mexicana. La linealidad de los modelos se demostró cuantitativamente en la validación interna mediante un valor del coeficiente de determinación muy cercano de la unidad; asimismo, por medio del ANDEVA, en el que el modelo lineal resultó altamente significativo, y la representación gráfica de los datos experimentales, que es una demostración cualitativa de la linealidad. Los residuales presentaron una distribución aleatoria cercana al cero.

La capacidad para estimar la concentración de HTP de los modelos se evaluó en la validación externa mediante las pruebas $t$ de Student y de 
correlación. Con la primera se demostró que no hay diferencias significativas entre los datos estimados con los modelos y los valores de referencia que se omitieron en la construcción y calibración de los modelos; y la segunda evidenció que hay un alto grado de asociación entre ambos conjuntos de datos. Además, los límites de detección y de cuantificación variaron en función del tipo de disolvente empleado (efecto matricial), siendo ambos valores más altos en los calibrados con éter etílico que con hexano, lo cual coincide con las ordenadas al origen en los modelos. Todos los indicadores estadísticos usados demuestran la robustez de los modelos propuestos y son la base para la evaluación de calibraciones lineales.

\section{REFERENCIAS}

Barwick V. (2003). Preparation of calibration curves. A guide to best practice. National Measurement System Valid Analytical Measurement (VAM) Programme, Department of Trade and Industry, UK [en línea]. https://www.lgcgroup.com/LGCGroup/media/PDFs/ Our\%20science/NMI\%20landing\%20page/Publications $\% 20$ and $\% 20$ resources/Guides/Calibration-curveguide.pdf 04/05/2016

Cuadros-Rodríguez L., Gámiz García L. y Almansa López E. (2001). Calibration in chemical measurement processes: I. A methodological approach. Trac-Trend. Anal. Chem. 20 (4), 195-206.

DOI: 10.1016/S0165-9936(00)00093-5

Danzer K. y Currie L.A. (1998). Guidelines for calibration in analytical chemistry. Part I. Fundamentals and single component calibration. IUPAC recommendations. Pure Appl. Chem. 70 (4), 993-1014.

DOI: $10.1351 /$ pac199870040993

Dost K. e Ideli C. (2012). Determination of polycyclic aromatic hydrocarbons in edible oils and barbecued food by HPLC/UV-Vis detection. Food Chem. 133 (1), 193-199. DOI: 10.1016/j.foodchem.2012.01.001

Fernández L., Rojas N., Roldan T., Ramírez M., Zegarra R. y Reyes R. (2006). Manual de técnicas de análisis de suelo aplicadas a la remediación de sitios contaminados. Secretaria de Medio Ambiente y Recursos Naturales-Instituto Nacional de Ecología-Instituto Mexicano del Petróleo, Ciudad de México, 180 pp.

Marqués F. (2010). Estadística descriptiva a través de Excel. Alfaomega, Ciudad de México, 288 pp.
Masschelei-Kleiner L. (2004). Los solventes. Centro Nacional de Conservación y Restauración, Santiago de Chile, 142 pp.

Mayer D.G. y Butler D.G. (1993). Statistical validation. Ecol. Model. 68 (1-2), 21-32.

DOI: $10.1016 / 0304-3800(93) 90105-2$

Newcombe R.G. (1998). Two-sided confidence intervals for the single proportion: Comparison of seven methods. Statist. Med. 17 (8), 857-872. DOI: 10.1002/ (SICI)1097-0258(19980430)

Raposo F. (2016). Evaluation of analytical calibration based on least-squares linear regression for instrumental techniques: A tutorial review. Trends Anal. Chem. 77, 167-185. DOI: 10.1016/j.trac.2015.12.006

Santos R.G., Loh W., Bannwart A.C. y Trevisan O.V. (2014). An overview of heavy oil properties and its recovery and transportation methods. Braz. J. Chem. Eng. 31 (03), 571-590.

DOI: $10.1590 / 0104-6632.20140313 \mathrm{~s} 00001853$

SEMARNAT (2013). Norma Oficial Mexicana NOM138-SEMARNAT/SSA1-2012. Límites máximos permisibles de hidrocarburos en suelos y lineamientos para el muestreo en la caracterización y especificaciones para la remediación. Secretaría de Medio Ambiente y Recursos Naturales. Diario Oficial de la Federación, México, 10 de septiembre.

Shrivastava A. y Gupta V.B. (2011). Methods for the determination of limit of detection and limit of quantification of the analytical methods. Chron. Young Sci. 2 (1), 21-25. DOI: 10.4103/2229-5186.79345

Valcárcel M. y Cárdenas M.S. (2000). Automatización y miniaturización en química analítica. Sringer-Verlag Ibérica, Barcelona, 290 pp.

Van Loco J., Elskens M., Croux C. y Beernaert H. (2002). Linearity of calibration curves: Use and misuse of the correlation coefficient. Accred. Qual. Assur. 7 (7), 281 285. DOI: $10.1007 / \mathrm{s} 00769-002-04787-6$

Vogt F., Tacke M., Jakusch M. y Mizaikoff B. (2000). A UV spectroscopic method for monitoring aromatic hydrocarbons dissolved in water. Anal. Chim. Acta 422 (2), 187-198. DOI: 10.1016/S0003-2670(00)01065-5

Wang J.G., Zhan X.H., Liang J.R., Zhou L.X., Lin Y.S. y Wong W.C. (2011). A novel method for the determination of total hydrocarbon in the hydrocarbon mixturecontaminated soil. J. Biorem. Biodegrad. 2, 1-6. DOI: 10.4172/2155-6199.S2-001

Weisberg S. (2005). Applied linear regression. 3rd ed. John Wiley and Sons, Hoboken, USA, 310 pp. 